\title{
Incorporación de nuevas estrategias en el aula. Relación entre el impacto académico y el rol asumido por el docente.
}

María del Carmen Maurel ${ }^{1}$, Liliana Raquel Cuenca Pletsch ${ }^{1}$ y Nidia Dalfaro ${ }^{1}$

Resumen

En la Facultad Regional Resistencia de la Universidad Tecnológica Nacional, se vienen desarrollando, desde el año 1995, cambios metodológicos en relación con los procesos de enseñanza y de aprendizaje. Los cambios a los que se aluden se pueden clasificar en la categoría incorporación de nuevas estrategias en el aula. En el presente trabajo se pretende mostrar que, independientemente de la estrategia a incorporar y el nivel de institucionalización de la propuesta, una variable importante del impacto académico en dicha incorporación es el rol asumido por el/los docentes intervinientes en la misma.

En este artículo se revisan diferentes experiencias académicas, el rol asumido por el docente en ellas y los resultados académicos de los estudiantes involucrados en las mismas. A partir de estos resultados, se analizan algunas exigencias y demandas para la práctica de los docentes universitarios. Se reconoce que estas prácticas pedagógicas obedecen al compromiso de la institución por asumir las problemáticas académicas de los estudiantes y realizar una revisión del paradigma de la formación impartida.

El análisis se desarrolló sobre la base de información cuanti-cualitativa obtenida a través de un relevamiento en nueve asignaturas pertenecientes a las tres carreras de Ingeniería que se desarrollan en la Facultad: Ingeniería Electromecánica, Ingeniería en Sistemas de Información e Ingeniería Química. Dicho relevamiento se realizó a partir de entrevistas a los docentes involucrados y consultas al Sistema de Gestión Académica de la Facultad (Sysacad), además del análisis de las planificaciones. 
Palabras Clave: innovación- rol docente- resultados académicos

\section{Abstract}

Since 1995, the Resistencia Regional Faculty of the National Technological University has been developing methodology changes in relation to teaching and learning. Such changes can be classified into the category of incorporation of new strategies in the classroom. This paper intends to show that, regardless of the strategy to incorporate and the proposal institutionalization level, an important variable of academic impact in such incorporation is the role assumed by the teachers involved.

This paper reviews different academic experiences, the role assumed by the teacher and the academic performance of the students involved. From these results, some requirements and demands for the practice of university teachers are discussed. It is known that these pedagogical practices are due to the institution commitment of assuming students' academic issues and reviewing the education paradigm.

The analysis was developed based on quantitative and qualitative information obtained through a survey in nine subjects from the three Engineering careers: Electromechanical Engineering, Information Systems Engineering and Chemical Engineering. This survey was based on interviews with the teachers involved and inquiries to Academic Management System of the Faculty (Sysacad), apart from curriculum plan analysis.

Keywords: innovation - teacher role - academic results. 


\section{Introducción}

El presente trabajo tiene como objetivo mostrar que, independientemente de la estrategia pedagógica a incorporar y el nivel de institucionalización de la propuesta, una variable importante en el impacto académico de una estrategia pedagógica es el rol asumido por el/los docentes intervinientes en la misma.

Todo indica que para transformar la enseñanza de la ingeniería es indispensable cambiar de estrategia, es decir modificar el modo tradicional de pensar y de hacer las reformas e innovaciones que involucran a los docentes. Aún cuando se actualicen las propuestas curriculares, se implementen programas de mejora de la enseñanza, se fomente la equidad y la calidad, si no se reconoce en los docentes el factor central de cambio, éste no tendrá lugar. Es necesario, en consecuencia, lograr reformas significativas, efectivas y ante todo sostenibles en las prácticas profesionales y culturas de trabajo de los docentes.

Cuenca Pletsch y Maurel (2011) afirman que "la flexibilidad en las interacciones, la posibilidad de revisar los debates y las producciones y el desplazamiento del docente desde los ámbitos formales de educación presencial hacia ámbitos virtuales, aunque también formales, tiene un impacto positivo en el rendimiento estudiantil" (p.338).

La mayoría de los estudios relacionados con el rendimiento académico, se centran en aspectos relacionados con el alumno. Según los estudios realizados en la Facultad Regional Resistencia, un porcentaje minoritario de los estudiantes muestra consistencia cuando se analizan las estrategias que utilizan para la apropiación y retención de los contenidos mediante técnicas que son más adecuadas y que presuponen mejores rendimientos académicos. La mayoría, al cabo de seis meses de iniciados sus estudios superiores, manifiesta emplear estrategias rutinarias y memorísticas, por lo que se puede suponer que dichos estudiantes tendrán mayores dificultades para abordar estudios universitarios; lo cual podrá ser significativo a la hora de analizar su rendimiento académico. (Dalfaro, Maurel y Soria, 2009).

Resulta imperioso, entonces, girar la mirada hacia el otro polo de la relación educativa. Después de dos décadas de reformas educativas con resultados relativos, es necesario aceptar algo que parece obvio pero no ha sido adecuadamente incorporado a las políticas institucionales: para cambiar la educación es necesario hacerlo con los docentes. A ellos se les reconoce un papel clave en las reformas educativas por razones que aparentemente son contradictorias y que en (Fullan, 1993) se resume muy acertadamente "la formación docente tiene el honor de ser, simultáneamente, el peor problema y la mejor solución en educación”. 


\section{Consideraciones generales sobre el rol docente.}

Duque y Celis (2012) resumen investigaciones referidas a las características que deben acreditar los docentes para una docencia de calidad y que tienen que ver con: comprensión disciplinar; conocimiento adicional del objeto de estudio necesario para poder enseñarlo (visiones de la disciplina, su historia, conexión con otras); conocimiento del diseño curricular; conocimiento pedagógico sobre lo que se enseña o sello pedagógico de la disciplina (creencias y concepciones de los estudiantes sobre el objeto de estudio, estrategias que facilitan el aprendizaje, empleo de apoyos tecnológicos para potenciar el proceso, estrategias para monitorear y evaluar el progreso del estudiante y modelos sobre cómo se aprende la disciplina). También deben tener compromiso con altos logros en la enseñanza, respeto por los estudiantes, su desarrollo y empoderamiento; compromiso con el desarrollo de comunidades de aprendizaje de alumnos y profesores; y compromiso con una reflexión continua y evaluación del proceso de enseñanza y su realimentación.

Un estudio realizado en la Argentina (Cataldi et al., 2004) buscó determinar el perfil del docente universitario desde la óptica estudiantil. Se trabajó mediante la aplicación de una encuesta a una muestra de 200 alumnos ingresantes a la Facultad de Ingeniería de la Universidad de Buenos Aires. Éstos debían indicar las 5 características que deseaban para sus docentes y las 5 que no deseaban. Las más valoradas fueron: que sea justo $(80 \%)$, paciente $(75 \%)$, claro en sus exposiciones $(70 \%)$, que explique las veces que sea necesario $(68 \%)$, que se interese por sus alumnos (65\%) y que tenga "buena onda" (53\%). Las características que no deseaban en sus docentes fueron: que sepa mucho pero no sepa explicar (75\%), que sea soberbio $(73 \%)$, que fume en clase $(68 \%)$, que se moleste si tiene que repetir (65\%), que sus actitudes sean de superioridad (48\%) y que abuse de su poder (45\%). Cabe destacar que estos resultados fueron refrendados y confirmados mediante las encuestas respondidas por los alumnos de la Facultad Regional Resistencia, durante los años 2010 a 2012.

Los trabajos referidos refuerzan la importancia que el alumno otorga a la calidad humana y profesional del docente. De él se espera que se convierta en facilitador del aprendizaje sin renunciar a su papel de maestro, abriendo otras fuentes de información para el estudiante, estimulando el trabajo en equipo y los valores sociales, sin restarle posibilidades al surgimiento individual, considerando que la educación no sólo es un hecho individual, sino ante todo social. Debe conocer los problemas del contexto social, con el fin de tener una mejor visión para orientar el trabajo del estudiante atendiendo a las necesidades y problemas reales del mercado laboral.

Los estudiosos del tema concluyen que los aspectos que destacan en el perfil deseado del docente universitario son de índole social, y de transposición del saber. 
En este sentido las instituciones deberían considerar, en forma prioritaria, en las evaluaciones del docente, aspectos de la interacción profesor- alumno tales como el buen trato y el interés hacia los estudiantes y no sólo habilidades de índole pedagógica.

\section{Experiencias académicas seleccionadas.}

A los fines de esta presentación se explicitan nueve experiencias de cátedras pertenecientes a las tres carreras de Ingeniería que se desarrollan en la Facultad: Ingeniería Electromecánica (IEM), Ingeniería en Sistemas de Información (ISI) e Ingeniería Química (IQ). El relevamiento de la información se realizó a través de entrevistas a los docentes, análisis de las planificaciones, encuestas a los alumnos y consultas al sistema académico de la Facultad (Sysacad).

Para una mejor descripción las hemos clasificado en tres categorías: experiencias de integración de contenidos, experiencias de incorporación de espacios virtuales en la enseñanza y experiencias de incorporación de nuevos recursos en la enseñanza.

\subsection{Experiencias de integración de contenidos.}

En esta categoría hemos encuadrado a las siguientes cátedras: Sistemas y Organizaciones (Integradora de ISI), Integradora I (Integradora de IEM) e Integradora I (integradora de IQ).

Este grupo de cátedras comparten la filosofía y las estrategias abordadas en los lineamientos curriculares de la UTN, que establecen un modelo de trabajo que integra los temas o contenidos con un abordaje teórico-práctico de manera conjunta, a partir del planteo de situaciones problematizadoras que integren los contenidos que se dictan simultáneamente en las cátedras del mismo nivel. Se utilizan estrategias pedagógicas que contribuyen a resignificar el conocimiento mediante la identificación y solución de problemas ingenieriles propios de la especialidad. Se proponen actividades a desarrollar por los alumnos en forma individual y grupal, según los temas. En algunos casos se debe presentar un informe de la actividad realizada.

En estas asignaturas los docentes deben promover la autogestión del aprendizaje, siendo su rol, fundamentalmente, orientador. En relación a los trabajos, el equipo docente define: objetivos, datos a conseguir, actividades a desarrollar, resultados a obtener, bibliografía básica a utilizar. Ayudan a identificar problemas de la carrera en cuestión y orientan para encontrar las posibles soluciones. En caso de Visitas a industria: preparan guía con objetivos, información a relevar, resultados a obtener, etc.

En el cierre de cada tema se presentan las conclusiones de las actividades realizadas, se rescatan los conceptos que se integraron, cómo fueron integrados, a qué área del conocimiento pertenecen, con la participación activa de los alumnos mediante opiniones y preguntas. 


\subsection{Experiencias de incorporación de espacios virtuales en la enseñanza.}

En este apartado se encuadran las cátedras: Sistemas Operativos ( $2^{\circ}$ año de ISI), Química Orgánica ( $2^{\circ}$ año de IQ) e Ingeniería de Software ( $4^{\circ}$ año de ISI). Si bien las tres tienen en común el uso o incorporación del espacio virtual como complemento de las clases presenciales, no todas hacen el mismo uso del espacio virtual.

En Sistemas Operativos se implementa el campus virtual como un medio para mejorar el contacto alumno-profesor y para hacer disponible, además del material utilizado en las clases, material digital elaborado en otras instituciones universitarias, artículos de investigación, links a sitios con material de estudios, audios de clase, videos. En lo relacionado con las clases de problemas y de laboratorios, se organiza a los alumnos en grupos, cada uno de los cuales debe resolver y subir al campus determinado problemas de las diferentes guías. Por cada grupo y guía de prácticos se habilita un foro, en el cual los estudiantes deben resolver los problemas en forma colaborativa, subir las propuestas de solución y posteriores correcciones surgidas del intercambio con los docentes y los compañeros de curso. De esta forma, queda disponible en el espacio virtual una versión corregida y correcta de cada problema y la discusión generada respecto de los conceptos teóricos que sustentan la resolución de la práctica. También se habilitan cuestionarios para que los alumnos se autoevalúen antes de los exámenes parciales.

Química Orgánica, por su lado, le ha asignado al espacio virtual el significado de un repositorio de materiales. En él el alumno dispone de series de ejercitaciones, guías de trabajos prácticos, material de organización de la cátedra, material para los seminarios de los laboratorios y para el desarrollo de los seminarios de orientación teórica. Las clases presenciales se desarrollan con el formato de seminarios, durante los cuales se exponen y debaten los temas de teoría, al cual previamente los alumnos pueden acceder en el campus. Se trata de lograr un debate dinámico entre docentes y alumnos, ya que desde el primer día de clases disponen de las guías que los ayudarán a organizar el estudio de cada tema del programa. La lectura previa es un requisito para el debate en clases.

En el caso de Ingeniería de Software, el equipo docente trabaja el espacio virtual con acento en la parte práctica. Se enumeran para cada unidad teórica, los objetivos, contenidos involucrados, bibliografía básica y se sube el práctico a desarrollar. Las guías de trabajos prácticos con formato pdf., para que el alumno la pueda bajar e imprimir. Como correlato de la guía se genera la tarea con la consigna y el espacio habilitado para subirla y ser evaluada por el docente de práctica. También se generan espacios de consulta sobre las actividades prácticas y espacios de información organizativa de la cátedra (fechas de parciales, recuperatorios, situación de los alumnos, etc.).

\subsection{Experiencias de incorporación de nuevos recursos en la enseñanza}

En este grupo hemos incluido a las cátedras: Física II ( $2^{\circ}$. año de IEM), Calidad y Productividad ( $4^{\circ}$ año de IEM) y Biotecnología ( $4^{\circ}$ año de IQ). Las tres comparten el haber incorporado una serie de recursos innovadores o no tradicionales; aunque 
la variedad y combinación que ha utilizado cada una de ellas las hace muy diferente una de otra.

En Física II, se desarrollan los temas a partir de una situación problemática de la vida real, tratando de establecer un dialogo con los alumnos con el fin de identificar el problema sobre el cual se trabajará, para luego avanzar, de manera formal, si fuera necesario con el apoyo de desarrollos matemáticos sencillos, haciendo énfasis en los conceptos físicos fundamentales involucrados y sus aplicaciones a la tecnología. En las clases teóricas se explicitan todos los conceptos requeridos para el desarrollo de los trabajos prácticos, resolución de problemas y prácticas de laboratorio; permitiendo que el alumno razone cada tema. En las clases de problemas se trabaja de manera grupal con guías de problemas propuestos. En las clases de laboratorio los alumnos trabajan en comisiones fijas de no más de cinco integrantes y cuentan con una guía de trabajo. Se trabaja con dataloggers y se incorporaran experiencias de laboratorios virtuales en algunos temas, como es el caso de campo eléctrico y potencial, circuitos eléctricos y óptica geométrica y física, dependiendo de la disponibilidad de las PC.

En el caso de Calidad y Productividad; en la introducción de cada uno de los temas se repasan algunas de las aplicaciones más características de los contenidos y se comprueban los conocimientos que tienen los alumnos sobre el tema a desarrollar, con el fin de seleccionar posteriores actividades de profundización. Si se trata de la continuación de un tema iniciado con anterioridad se hace un repaso o enlace antes de continuar el desarrollo. Se trabaja de manera combinada la exposición, la demostración y ejemplificación. Los docentes buscan promover en todo momento la participación de los alumnos mediante opiniones, análisis, ejemplificaciones, debates, inducciones. Al finalizar un eje de contenidos se realiza una reflexión y puesta en común de conclusiones conducida por el profesor. Los contenidos son afianzados a través de la realización de trabajos prácticos, normalmente en pequeños grupos. El docente resuelve en clase un ejercicio modelo donde se aplican los contenidos vistos previamente a un caso concreto vinculado con la especialidad y luego los alumnos resuelven los que se encuentran en la guía de trabajos prácticos. También, como integración de teoría y práctica, se les pide a los alumnos la elaboración de un plan de aseguramiento de la calidad, buscando de esa manera que el alumno adquiera las habilidades básicas para implementar la gestión de procesos en base a normas de calidad.

En Biotecnología se plantean una diversidad de estrategias: de tratamiento de la información (exposición, exposición dialogada, elaboración de esquemas, y/o mapas conceptuales), de socialización (trabajo en equipo, simposio, mesa redonda, debate dirigido), estrategias personalizadas (método de trabajo por proyecto), estrategias creativas (torbellino de ideas) y estrategias de trabajo con tecnologías de la información y comunicación (uso de recursos multimedia y de sitios de Internet). Las clases teóricas son conducidas por la profesora a cargo de la cátedra, contando con la apoyatura de un auxiliar docente. Se incorporan docentes invitados o egresados 
de la casa cuando se considera oportuno. Las prácticas de laboratorio son guiadas por el auxiliar docente, con la apoyatura de la profesora. Se utiliza el aula virtual como medio de comunicación con el alumno, para todas las actividades propuestas.

\section{Resultados académicos obtenidos por los alumnos.}

Se presentan a continuación, para cada grupo de experiencias, los resultados académicos de los últimos cinco años.

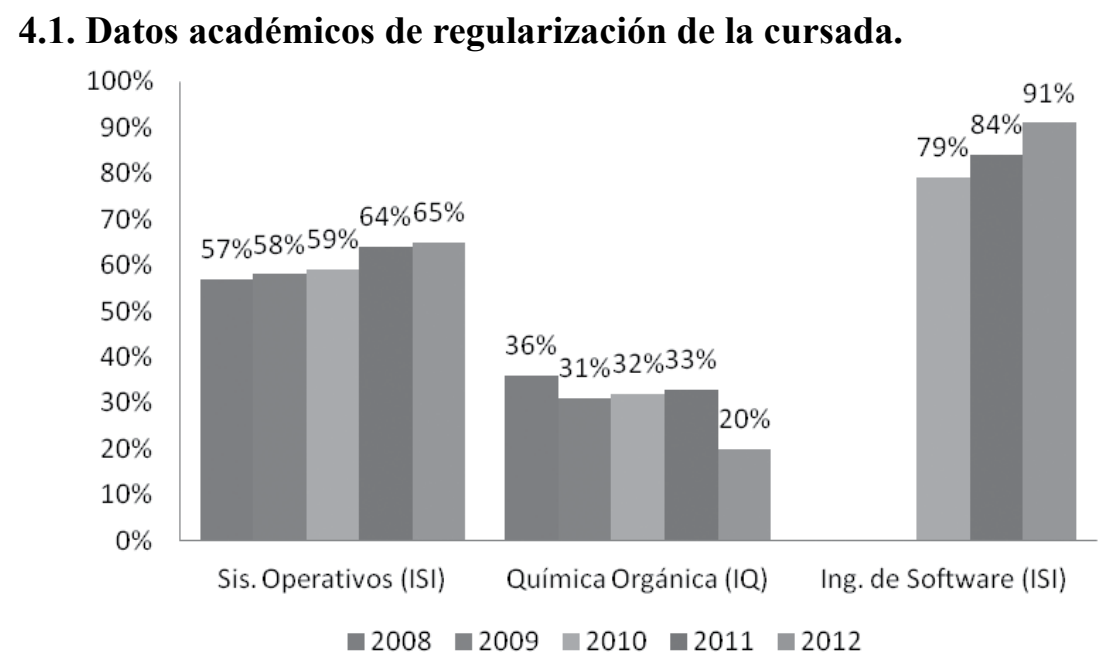

Fig.1. Experiencias de integración de contenidos

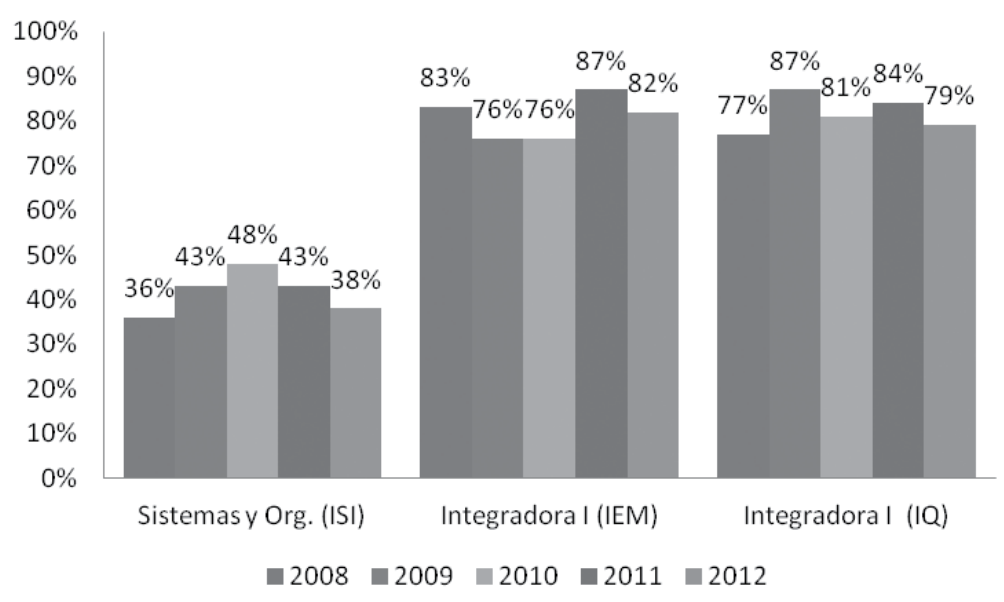

Fig.2. Exp. de incorporación de esp. virtuales en la enseñanza. 


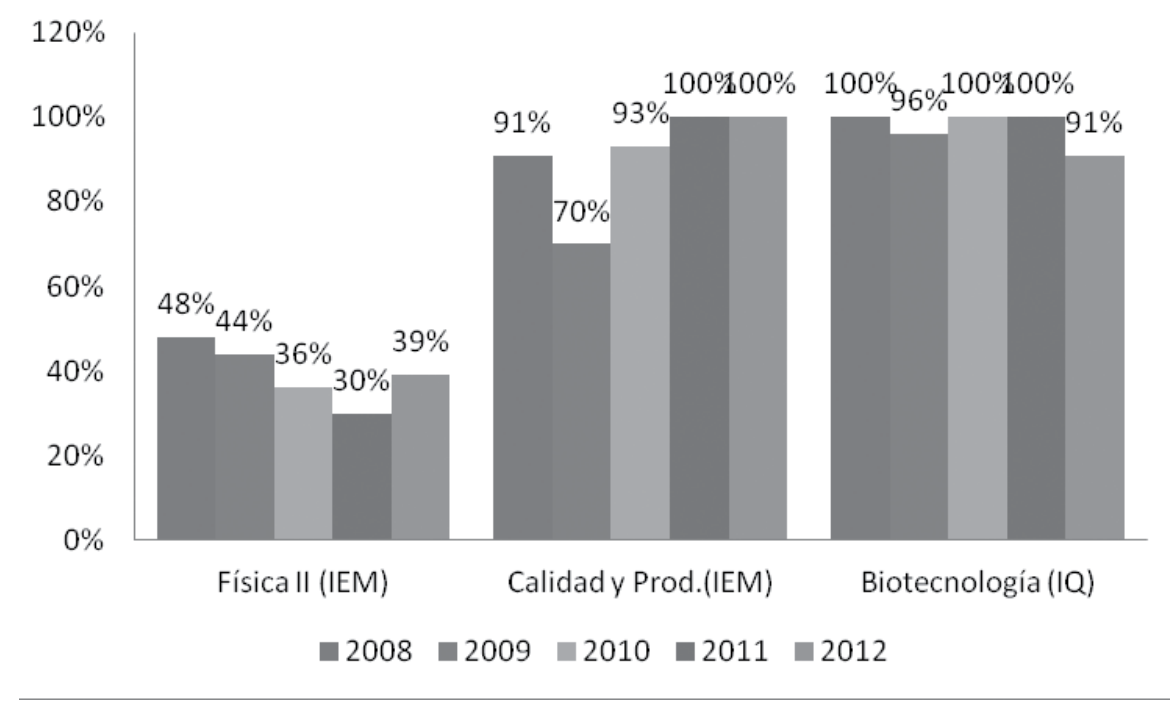

Fig.3. Experiencias de incorporación de nuevos recursos en la enseñanza

\subsection{Datos académicos de aprobación de la materia.}

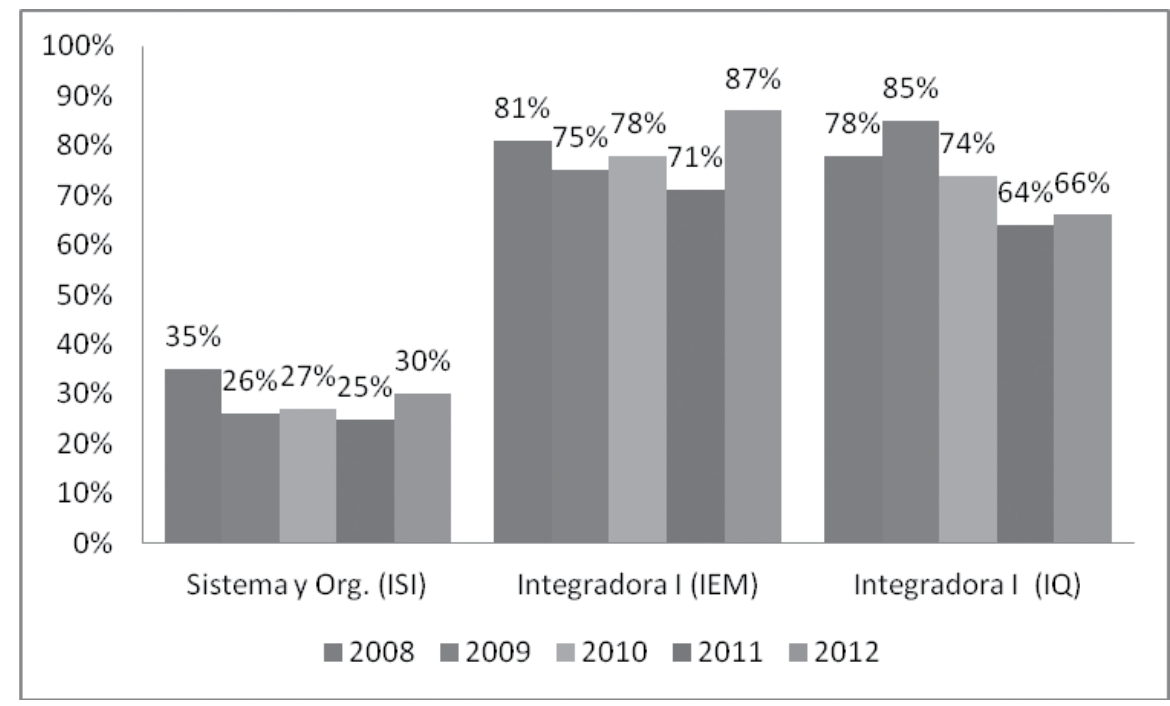

Fig.4. Exp. de integración de contenidos 


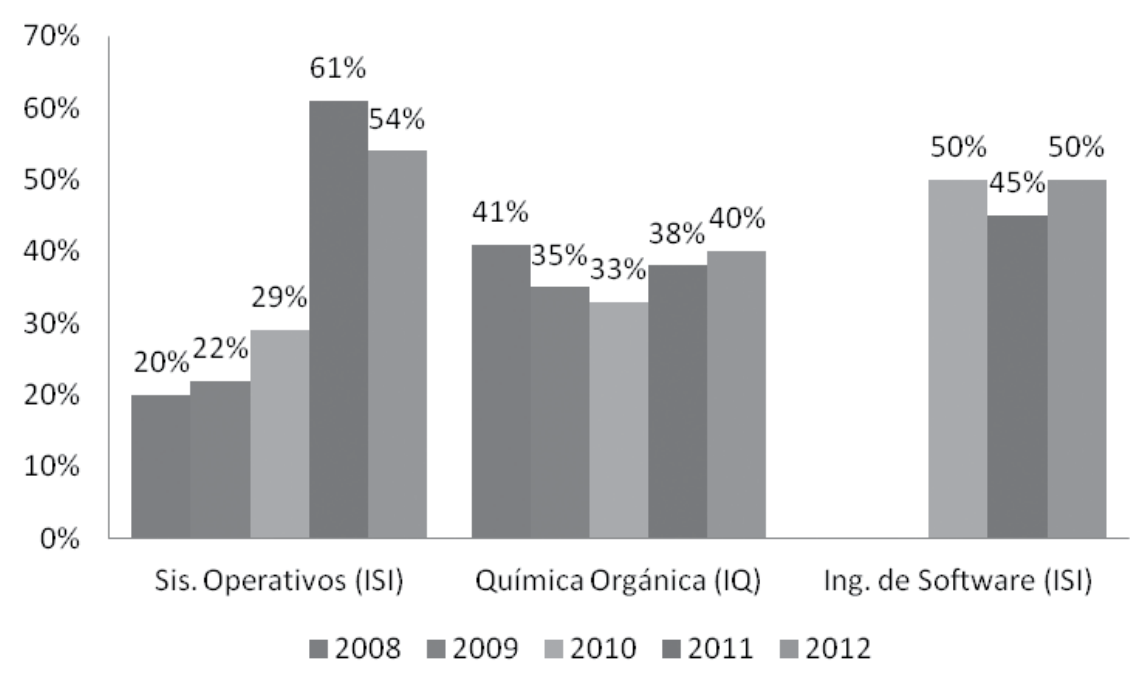

Fig.5. Exp. de incorporación de espacios virtuales en la enseñanza

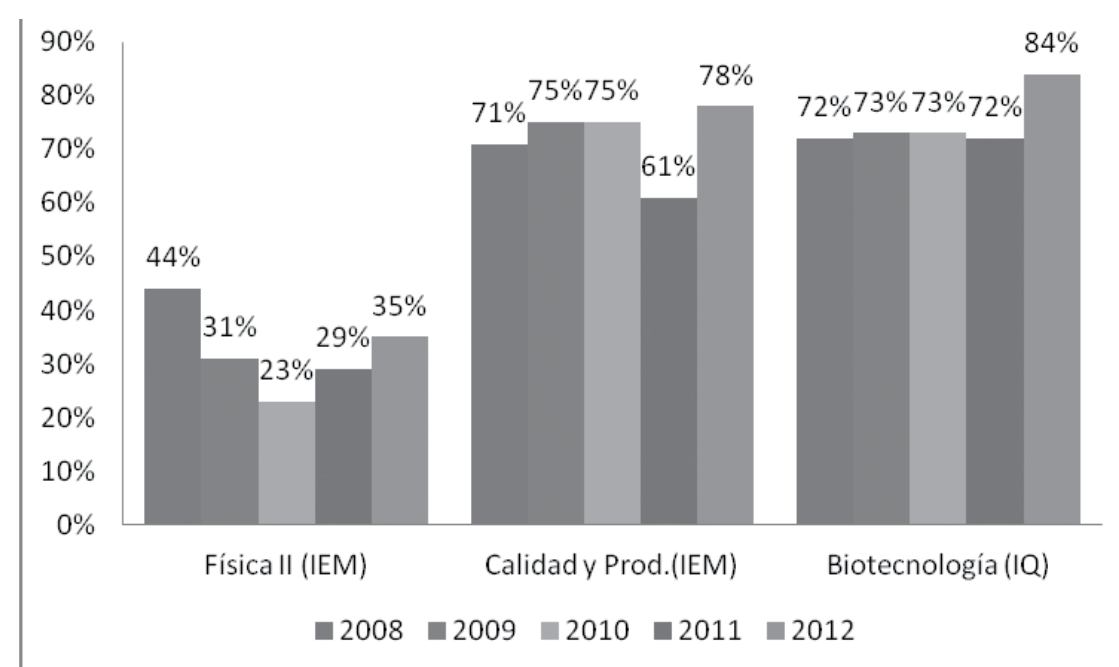

Fig.6. Experiencias de incorporación de nuevos recursos en la enseñanza.

Es posible observar diferentes rendimientos académicos, por encima o por debajo del $50 \%$, entre asignaturas que adoptan idénticas estrategias docentes. Estas diferencias estarían indicando que la estrategia por sí sola no garantiza el éxito ni el fracaso. Se orientó entonces la búsqueda de respuestas en las entrevistas con los docentes y en las encuestas que los estudiantes completan en el marco del programa de carrera académica. Ambas reflejan claramente, y en consonancia con el marco teórico, que los resultados están íntimamente vinculados con el rol que asumen los docentes en el proceso de formación. 5. 


\section{Conclusiones}

Los resultados académicos expuestos se corresponden con el rol asumido por el equipo docente de la cátedra al incorporar nuevas estrategias o innovaciones para el desarrollo de la materia. En los casos en que el equipo docente asume el rol de guía, se interesa por el aprendizaje de los alumnos, tiene un trato cordial, fomenta la comunicación y la participación; los resultados académicos son altamente positivos, independientemente de la estrategia innovadora que se utilice.

Por el contrario, en las cátedras en las que, la incorporación de estrategias innovadoras no se condice con mejores rendimientos; aparecen ciertas características en el equipo docente que justificarían dichos resultados. En efecto, según opinión de los alumnos, estos equipos no logran una interacción positiva con los estudiantes. Los docentes se presentan como muy exigentes y no hay una adecuación entre la cantidad de contenido desarrollada y el tiempo asignado a la materia en el plan de estudio.

En la actualidad hay consenso en cuanto que el ingeniero debe saber hacer, lo cual no surge de la mera adquisición de conocimientos sino que es el resultado de la puesta en acción de una compleja estructura de conocimientos, habilidades, destrezas que requiere ser reconocida expresamente en el proceso de aprendizaje para que la propuesta pedagógica incluya las actividades que permitan su desarrollo.

Resulta claro que es necesario establecer, como política institucional, la mejora en los procesos de enseñanza-aprendizaje. Esta mejora implica, necesariamente, innovación e incorporación de nuevas estrategias de enseñanza, lo cual, a su vez, requiere de un real involucramiento de los docentes. Las carreras de Ingeniería requieren de profesores que sepan hacer, que conozcan la disciplina, que tengan claro cómo se aprende el saber que deben transmitir, que estén comprometidos con el aprendizaje de sus alumnos y que estén dispuestos a ser parte del cambio. 


\section{Referencias}

Cataldi, Z.; Lage, F. (2004). Informática y medios audiovisuales. Revista de Informática educativa y medios audiovisuales. Recuperado de: http://www.fi.uba. ar/laboratorios/lie/Revista/Articulos/010103/A3oct2004.pdf

Cuenca Pletsch, Liliana; Maurel, María del Carmen. (2011). El Blended Learning: un medio para promover el aprendizaje colaborativo. $1^{\circ}$ Jornada de Enseñanza de la Ingeniería - JEI 2011 (pp 338-345), ISBN: 978-950-42-0139-7. Buenos Aires, Argentina.,

Dalfaro, Nidia A.; Maurel, María del Carmen y Soria, Fernando H. (2009). "Atribuciones Causales de Docentes y Alumnos de Ingeniería acerca del Desgranamiento Temprano" En: Encuentro La Universidad como Objeto de Investigación. Universidad Nacional de Córdoba. Argentina.

Duque, M; Celis, J (2012). Educación en Ingeniería para la ciudadanía, la innovación y la competitividad en Iberoamérica. ASIBEI, Bogotá, Colombia, pp 44-45.

Fullan, M. (1993). Change forces: probing the depths of Educational Reform. London, the Falmer Press. 